\title{
Autonomous Sensory Meridian Response self-reporters showed higher scores for cognitive reappraisal as an emotion regulation strategy
}

\author{
Ricardo Morales ${ }^{1,2}$, Daniela Ramírez-Benavides ${ }^{1}$, Mario Villena-Gonzalez ${ }^{\text {Corresp. 1,3 }}$ \\ 1 Escuela de Psicología, Pontificia Universidad Católica de Chile, Santiago, RM, Chile \\ 2 Center for Cognitive Neuroscience, Duke University, Durham, NC, United States \\ ${ }^{3}$ Centro de Estudios en Neurociencia Humana y Neuropsicología, Facultad de Psicología, Universidad Diego Portales, Santiago, RM, Chile \\ Corresponding Author: Mario Villena-Gonzalez \\ Email address: mvillena@ug.uchile.cl
}

Background: Autonomous Sensory Meridian Response (ASMR) describes the experience of a pleasant tingling sensation along the back of the head, accompanied with a feeling of well-being and relaxation, in response to specific audio-visual stimuli, such as whispers, soft sounds, and personal attention. Previous works have assessed individual variations in personality traits associated with ASMR, but no research to date has explored differences in emotion regulation associated with ASMR. This omission occurred even when ASMR, a sensory-emotional experience, has been proposed to be located in a sound sensitivity spectrum as the opposite end of misophonia, a phenomenon associated with difficulties regulating emotions. The present work aimed to assess group differences between ASMR self-reporters and non-ASMR controls associated with emotion regulation strategies.

Methods: We used the validated Spanish version of the Emotion Regulation Questionnaire to assess individual differences in the use of cognitive reappraisal and expressive suppression. Results: Our results showed that participants who experience ASMR had higher scores in the cognitive reappraisal subscale of the emotion regulation questionnaire than the non-ASMR group. Conclusions: Individuals who experience ASMR reported higher use of cognitive reevaluation of emotionally arousing situations, suggesting more effectiveness in regulating emotions. Our finding further elucidates individual differences related to this experience, supporting that ASMR is a real psychophysiological phenomenon associated with other psychological constructs and has remarkable consequences in affective/emotional dimensions and general well-being. 


\section{Autonomous Sensory Meridian Response self-reporters \\ 2 showed higher scores for cognitive reappraisal as an \\ 3 emotion regulation strategy}

4

5 Ricardo Morales ${ }^{1,2}$, Daniela Ramírez-Benavides ${ }^{1}$, Mario Villena-Gonzalez ${ }^{1,3 *}$

6

$7 \quad{ }^{1}$ Escuela de Psicología, Pontificia Universidad Católica de Chile, Santiago, RM, Chile

$8{ }^{2}$ Center for Cognitive Neuroscience, Duke University, Durham, NC, USA

$9{ }^{3}$ Centro de Estudios en Neurociencia Humana y Neuropsicología. Facultad de Psicología,

10 Universidad Diego Portales, Santiago, RM, Chile.

Corresponding Author:

Mario Villena-Gonzalez*

Vicuña Mackenna 4860, Santiago, RM, CP: 7820436, Chile

Email address: mvillena@ug.uchile.cl 


\section{Abstract}

27 Background: Autonomous Sensory Meridian Response (ASMR) describes the experience of a pleasant tingling sensation along the back of the head, accompanied with a feeling of well-being and relaxation, in response to specific audio-visual stimuli, such as whispers, soft sounds, and

30

31

\section{7} personal attention. Previous works have assessed individual variations in personality traits associated with ASMR, but no research to date has explored differences in emotion regulation associated with ASMR. This omission occurred even when ASMR, a sensory-emotional experience, has been proposed to be located in a sound sensitivity spectrum as the opposite end of misophonia, a phenomenon associated with difficulties regulating emotions. The present work aimed to assess group differences between ASMR self-reporters and non-ASMR controls associated with emotion regulation strategies.

Methods: We used the validated Spanish version of the Emotion Regulation Questionnaire to assess individual differences in the use of cognitive reappraisal and expressive suppression.

Results: Our results showed that participants who experience ASMR had higher scores in the cognitive reappraisal subscale of the emotion regulation questionnaire than the non-ASMR group.

Conclusions: Individuals who experience ASMR reported higher use of cognitive reevaluation of emotionally arousing situations, suggesting more effectiveness in regulating emotions. Our finding further elucidates individual differences related to this experience, supporting that ASMR is a real psychophysiological phenomenon associated with other psychological constructs and has remarkable consequences in affective/emotional dimensions and general well-being. 
55

56

57

\section{Introduction}

Autonomous Sensory Meridian Response (ASMR) describes the experience of a pleasant tingling sensation along the back of the head and neck region, at times spreading to other areas of the body, in response to specific visual and auditory stimuli (Barratt \& Davis, 2015). Usually, ASMR is triggered by whispers, soft sounds made with the fingers on a surface, and personal attention from someone with an affectionate disposition (Barratt, Spence, \& Davis, 2017; Fredborg, Clark, \& Smith, 2017; Poerio, Blakey, Hostler, \& Veltri, 2018).

This non-scientific term "ASMR" was coined just in 2010 (del Campo \& Kehle, 2016; Morris, 2018). During the last decade, social networks have made it possible to publicize this phenomenon and quickly disseminate different personal reports on this experience, along with the creation of videos ("ASMR videos") that simulate or accentuate the stimuli to trigger the sensation in people who watch/listen to them (del Campo \& Kehle, 2016). Anecdotal accounts and early research have shown that ASMR seems to be experienced by only part of the population, and therefore some individuals do not experience it (Barratt \& Davis, 2015; del Campo \& Kehle, 2016). There are no studies to date about the prevalence of ASMR-capability in the general population; however, some studies have offered some estimates suggesting the phenomenon is widespread (Poerio, 2016; Roberts, Beath, \& Boag, 2020). ASMR's popularity on the internet supports how prevalent this phenomenon might be (Figure S.1).

Scientific investigation about this experience started even more recently, with the first peerreviewed paper on the topic published in 2015 (Barratt \& Davis, 2015). Since then, there has been a growing interest in deciphering this phenomenon's underlying psychological and biological basis (Figure S.2).

Some previous works have assessed individual variations in personality traits associated with ASMR. These works have shown that, compared with non-ASMR controls, ASMR self-reporters scored higher on openness to experience (Fredborg et al., 2017; McErlean \& Banissy, 2017), which is associated with curiosity about the world around them and propensity to have vivid fantasies or daydreams (John, Naumann, \& Soto, 2008). In line with these results, other works have shown ASMR self-reporters score higher on Fantasizing (McErlean \& Banissy, 2017), reflecting an ability to immerse oneself in a fictional reality (Davis, 1983). They also produced higher scores on the Curiosity subscale of the Toronto Mindfulness Scale (TMS), suggesting a greater interest in and openness to their own conscious experiences (Fredborg, Clark, \& Smith, 2018). Individuals with ASMR also showed higher scores on neuroticism, associated with increased self-consciousness ( Fredborg et al., 2017). Finally, ASMR self-reporters have shown to score higher on absorption (McErlean \& Osborne-Ford, 2020; Roberts, Beath, \& Boag, 2019), which is the ability to get deeply immersed with the current experience accompanied by loss of 
91

92

93

94

95

96

97

98

99

100

101

102

103

104

105

106

107

108

109

110

111

112

113

114

115

116

117

118

119

120

121

122

123

124

125

126

reflective awareness, and it has been previously linked to imagery, daydreaming, and openness to experience (Glisky, Tataryn, Tobias, Kihlstrom, \& McConkey, 1991). All these results together suggest that an increased tendency to have openness to conscious experience, fantasize, and imaginatively transposing oneself into a virtual reality may be a key feature of ASMR responders.

Despite the notorious and growing interest in the subject, no research has explored the individual variations of emotional-related traits associated with ASMR, excepting some works assessing the relation between empathy and ASMR (McErlean \& Banissy, 2017; Poerio et al., 2018). The lack of studies directly evaluating emotional-related traits has occurred even when the literature is continuously emphasizing that ASMR is a sensory-emotional experience rather than just a sensory one (Fredborg et al., 2018; Smith, Fredborg, \& Kornelsen, 2019; Smith, Fredborg, \& Kornelsen, 2017).

Previous works have revealed the emotional dimension of ASMR by evaluating self-report measures, neuroimaging, and physiology. For instance, according to the first study on the topic, $80 \%$ of the participants reported watching ASMR videos because it positively affects their mood, usually accompanied by a pleasant feeling of well-being and relaxation (Barratt \& Davis, 2015). With regard to the neuroimaging approach, activation in regions related to emotional arousal (dorsal anterior cingulate cortex, insula, and inferior frontal gyrus) has been shown using fMRI during tingling sensations (Lochte, Guillory, Richard, \& Kelley, 2018). Another study measured physiological responses comparing ASMR self-reporters and non-ASMR controls while watching ASMR videos. The results showed a significant reduction in heart rate and increased skin conductance levels in ASMR self-reporters compared with non-ASMR controls suggesting that ASMR is a complex emotional experience blending different emotional components such as relaxation and arousal (Poerio et al., 2018).

In line with these previous studies supporting ASMR as an emotional experience, ASMR has also been suggested to be related to another underreported condition known as misophonia, where familiar repetitive sounds, mostly sound produced by humans such as chewing and sniffing, may trigger negative physiological, emotional, and behavioral responses (Roberts et al., 2019; Rouw \& Erfanian, 2018).

Previous works have suggested that misophonia and ASMR might represent two ends of the same spectrum of sound sensitivity where human-generated sounds elicit pleasurable tingling sensation in case of ASMR and negative physical and emotional responses in case of misophonia (Barratt \& Davis, 2015; McErlean \& Banissy, 2018).

Previous works have shown that individual variations in the severity of misophonia symptoms positively correlate with emotional regulation difficulties (Cassiello-Robbins et al., 2020). Neuroimaging approaches to misophonia revealed abnormal functional connectivity between the 
127 anterior insular cortex (a hub of the salience network critical for interoception and emotion 128 processing) and brain regions responsible for the regulation of emotions (Kumar et al., 2017).

129 It is interesting to note that even when misophonia and ASMR have been proposed as opposite

130

131

132

133

134

135

136

137

138

139

140

141

142

143

144

145

146

147

148

149

150

151

152

153

154

155

156

157

158

159

160

161

162

poles on the same spectrum, they also have been reported to overlap and co-occur in the same individuals, but the experience may depend on the trigger stimuli or context (Roberts et al., 2019; Rouw \& Erfanian, 2018).

ASMR has also been widely associated with another related sensory-emotional phenomenon known as frisson or music chills (del Campo \& Kehle, 2016). Frisson is a chill down the spine sensation that occurs while engaged in enjoyable music and other emotional experiences and is generally described as a pleasant sensation with an emotional load; Tears, gooseflesh, or piloerection could accompany it (Harrison \& Loui, 2014). Neuroimaging studies have shown that brain activation during ASMR showed similarities to patterns previously observed in music chills, associated with the striatal reward pathway and emotional arousal regions (Lochte et al., 2018; Salimpoor, Benovoy, Larcher, Dagher, \& Zatorre, 2011). These similarities can also be found in electrophysiological measures, in which both phenomena elicit an increase in skin conductance ( Poerio et al., 2018; Salimpoor et al., 2011).

Nonetheless, essential differences have been observed between these phenomena involving activation of the medial prefrontal cortex (mPFC). This region showed increased activation during ASMR tingling sensation, which has not been observed for music chills (Lochte et al., 2018). Activation of mPFC has been associated with higher scores in empathy and emotional regulation capabilities (Esménio et al., 2019; Tang, Tang, \& Posner, 2016), especially for reappraisal (Nelson, Fitzgerald, Klumpp, Shankman, \& Phan, 2015). In line with this, previous research has shown that ASMR self-reporters have higher Empathic Concern levels (McErlean \& Banissy, 2017). This trait has been previously associated with higher scores in cognitive reappraisal (Laghi, Lonigro, Pallini, \& Baiocco, 2018; Lockwood, Seara-Cardoso, \& Viding, 2014)

Taking this evidence into account, it would be logical to assume that the ability to feel ASMR could be associated with differences in some facet of emotional regulation, for instance, the regulation strategy deployed (suppression or reappraisal). However, there is no evidence about ASMR/non-ASMR group differences related to the emotional regulation abilities to date.

Individual differences in emotion regulation mechanisms can be investigated by studying the most common strategies: suppression and reappraisal (Gross \& John, 2003). Suppression is a form of response modulation in which the ongoing emotion-expressive behavior becomes inhibited. Reappraisal involves a cognitive reevaluation of the emotionally arousing situation to alter its emotional impact. Research on this topic has shown that using reappraisal is more effective and related positively to well-being, whereas using suppression is negatively related

PeerJ reviewing PDF | (2020:12:56562:2:0:NEW 15 Apr 2021) 
163 (Gross \& John, 2003; Morawetz, Alexandrowicz, \& Heekeren, 2017). Previous studies 164 demonstrated an association between reappraisal use and personality traits such as neuroticism 165 and openness to experience (Morawetz et al., 2017; Wang, Shi, \& Li, 2009). These same traits 166 have also been strongly associated with people who experience ASMR (Fredborg et al., 2017; 167 McErlean \& Banissy, 2017).

168 The present work aims to assess group differences between ASMR self-reporters and non-ASMR 169 groups associated with emotional regulation strategies. We hypothesized that people who experience ASMR, in the same way they seek to listen/watch ASMR stimuli to improve their positive affect and well-being, use an emotional regulation strategy that better provides effectiveness regarding well-being in different situations. We predicted that being the opposite extreme of misophonia (which is a condition related to difficulties in emotional regulation), people who experience ASMR will show greater cognitive reappraisal use than non-ASMR selfreported controls. This strategy has been positively associated with well-being and used by people who share personality traits common to ASMR groups. Finally, this prediction is also supported by the ASMR self-reporters' known capabilities regarding fantasy/absorption, which could facilitate the propensity to change the way they think about emotional situations.

We used the validated Spanish version of the Emotion Regulation Questionnaire (Cabello, Salguero, Fernández-Berrocal, \& Gross, 2013) to assess individual differences in the use of cognitive reappraisal and expressive suppression in ASMR self-reporters and non-ASMR controls.

This is the first study to examine whether ASMR self-reporters show differences associated with emotional regulation strategies compared with non-ASMR controls. Therefore, this work further elucidates individual differences related to this phenomenon and its remarkable consequences in affective/emotional dimensions and well-being.

\section{Materials \& Methods}

\subsection{Participants}

The total number of participants who answered the online survey was 177. From this total, only 69 reported not experiencing ASMR. One of these 69 participants answered the same response to all of the questions in the cognitive reappraisal and expressive suppression subscales. Due to this pattern of responses, we decided to exclude this participant's data from further analysis. Our sample consisted of 108 participants who report to experience ASMR and 68 participants who do not report to experience ASMR. All participants were native Spanish speakers. The matched samples' nationalities were mostly Chilean; 167 Chilean, 2 Argentinian, 3 Spanish, 2 Venezuelan, 1 Mexican, and 1 Peruvian. 
198 Due to the high proportion of participants who reported experiencing ASMR, we took a random

199

200

201

202

203

204

205

206

207

208

209

210

211

212

213

214

215

216

217

218

219

220

221

222

223

224

225

226

227

228

229

230

231

232

subsample of 68 participants from 108 who declared experiencing ASMR to attain similar sample sizes. The matched samples consisted of 136 volunteers; 68 participants in the ASMR group ( 51 female, mean age $=22.40, S D=3.32$ ) and 68 participants in the non-ASMR group (52 female, mean age $=23.81, S D=5.51)$. Groups did not differ in age $(t(134)=1.81, p=.07)$ nor in the proportion of male to female participants $\left(\chi^{\wedge} 2(1)<0.01, p=1\right)$. Analysis with the total sample of the participants can be found in the supplementary materials. The participants were not rewarded for participating in this study.

\subsection{Procedure}

Participants were recruited by an online invitation to participate in the present work. This invitation was spread through social media, specifically through Instagram and Facebook, posted in ASMR groups and also in groups non-related to ASMR (e.g., University students groups on Facebook). The invitation explained the study without giving away information related to ASMR to avoid bias towards people who might know and were interested exclusively in ASMR.

Participants were asked to complete a survey hosted in Google forms. This online survey included questions about personal information (age and gender) followed by the Spanish version of the Emotion Regulation Questionnaire (Cabello et al., 2013). Then, they were provided with the Spanish version of the ASMR-15 Score questionnaire (Roberts et al., 2019).

Afterward, participants were asked if they knew what Autonomous Sensory Meridian Response is. Regardless of the answer, they then were provided with a description and question about experiencing ASMR. Specifically, participants were provided with the following statement: “ASMR is defined as a pleasant sensation of 'tingles' across the back of the head and neck in response to specific visual and auditory stimuli." Then, participants were asked to answer the following question, "would you defined yourself as someone who experiences ASMR?" with a Yes or No response. We divided the ASMR self-reporters and non-ASMR groups based on this response. Additionally, we further asked the ASMR group to provide their favorite ASMR video link/channel and answer some additional questions about the frequency of watching ASMR videos/content and the main reason for watching them if that was the case (see supplementary material).

The protocol was approved by the Ethics Committee of Pontificia Universidad Católica de Chile (approval reference number: 190325015). All participants gave electronic informed consent following the Declaration of Helsinki. This study was conducted online, and participants completed the questionnaires in their own time in one sitting. 


\subsection{Materials}

235

\section{Emotion Regulation Questionnaire (ERQ)}

237 The ERQ is a self-report questionnaire that consists of 10-items assessing two different

238

239

240

241

242

243

244

245

246

247

248

249

250

251

252

253

254

255

256

257

258

259

260

261

262

263

264

265

266

267

268

269 regulation strategies. The first one is called cognitive reappraisal based on six items, and the second one is called expressive suppression based on four items. Cognitive reappraisal focuses on the person's attempts to change the emotional impact by changing how they think of the situation. Expressive suppression focuses on the person's attempts to hide or inhibit the expression of their emotions. The instruction was: "We would like to ask you some questions about your emotional life, in particular, how you control (that is, regulate and manage) your emotions. We are interested in two main aspects: on the one hand, your emotional experience, or how you feel the emotions; and on the other hand, the emotional expression or how you show emotions in the way you talk or behave. Although some of the following questions may seem similar, they differ in quite important ways. Please answer how much you agree or disagree with each of the questions below using the following response scale". Both subscales are measured with simple questions answered on a Likert scale ranging from 1 (totally disagree) to 7 (totally agree). The higher scores signify more usage of the strategy they are referring to in that specific question. In this case, the questionnaire used was the Spanish adaptation of the ERQ, which has been translated and validated for Spanish-speaking users, showing good internal consistency for both subscales; expressive suppression (Cronbach's alpha $=0.75$ ) and cognitive reappraisal (Cronbach's alpha $=0.79$ ) (Cabello et al., 2013). This questionnaire's internal consistency calculated from data of the present study is similar to those reported in Cabello et al., 2013, with a Cronbach's alpha $=0.76$ for cognitive reappraisal and Cronbach's alpha $=0.78$ for expressive suppression.

\section{ASMR-15 SCORE}

The ASMR-15 is a multidimensional self-report measure of ASMR propensity that consists of 15-items assessing four different characteristics of the phenomenon; sensation, relaxation, affect, and altered consciousness (Roberts et al., 2019). "Sensation" describes aspects of the location and physical sensation. "Relaxation" involves changes in arousal associated with calm and relaxation. "Affect" describes aspects of emotional experience. Finally, "Altered Consciousness" is associated with shifts in perception and awareness. In this questionnaire, five items are related to sensation, three related to relaxation, four related to altered consciousness state as a result of the ASMR experience, and three others are related to affect, specifically to changes in emotional states. Participants answer with a Likert scale ranging from 1 (totally disagree) to 5 (totally agree), where higher scores indicate a greater tendency to experience ASMR. We used this scale 
as a global corroboration that the selection of groups based on self-report was different on the different dimensions of the phenomenon.

The scale has a brief introduction: "This survey is looking at how certain stimuli affect you. Some individuals experience intense physical and emotional responses upon hearing particular sounds. These sensations and feelings can be pleasant or unpleasant. Sounds such as whispering, crackling, tapping, or scratching may produce particular experiences described below. Using the scale, please indicate your level of agreement with each statement, upon hearing any of these, or similar sounds. When I hear certain sounds, such as whispering, crinkling, tapping...”.

For this questionnaire, our research group translated the original instrument to a Spanish version, which showed good levels of internal consistency in the four subscales. The sensation subscale had a Cronbach's alpha $=0.81$, the relaxation subscale had a Cronbach's alpha $=0.88$, the affect subscale had a Cronbach's alpha $=0.80$, and the altered consciousness subscale had a Cronbach's alpha $=0.83$. The total score (the mean of the 15 items) had a Cronbach's alpha $=$ 0.90 .

\subsection{Statistics}

All the data analysis, processing, basic descriptive statistics, testing of assumptions, and comparison between means were performed using the R and RStudio software (Allaire, 2012). Reliability analysis of the subscales of the ERQ and ASMR-15 was made with the psych package (Revelle \& Revelle, 2015) and plots with the ggplot2 package (Valero-Mora, 2010).

To match the sample sizes between the ASMR and non-ASMR groups we randomly selected a subsample from the group with more participants, equal to the number of participants of the group with fewer participants. We repeated this procedure 1000 times to corroborate the replicability of the effect. To test if the ASMR and non-ASMR groups differ in age or male to female participants, we performed a t-test and a chi-square test, respectively. Subsequently, we used the Cronbach alpha coefficient to measure the internal consistency of the subscales of the ERQ and the ASMR-15. All of the comparisons between the ASMR and non-ASMR groups were performed with independent sample t-tests.

\section{Results}

Our main aim was to assess whether ASMR self-reporters show differences in emotional regulation strategies compared to the non-ASMR group. We performed individual comparisons between each of the subscales of the questionnaire. To maintain the experiment-wise error rate to $5 \%$, we set an alpha value of $2.5 \%$ (a significant $p$-value of .025).

\section{Normality and homogeneity of variance}


307 To test the assumptions of normality and homogeneity of variance, we use the Shapiro-Wilk and

308 Levene tests, respectively. In the Expressive suppression subscale, data from the ASMR group $309(W=0.98, p=.41)$, and data from the non-ASMR $(W=0.97, p=.07)$ showed a normal 310 distribution. In the Cognitive reappraisal subscale, both the ASMR group $(W=0.97, p=.11)$ and 311 the non-ASMR group ( $W=0.98, p=.37$ ) showed a normal distribution. The variance between 312 the ASMR and non-ASMR groups were similar in both the Expressive Suppression subscale $313(F(1,134)=2.31, p=.13)$ and in the Cognitive Reappraisal subscale $(F(1,134)=0.11, p=.76)$. 314 In neither group, participants with $\mathrm{z}$ scores bigger than 3 or lesser than -3 were detected; 315 accordingly, we didn't reject any case (Tabachnick \& Fidell, 2013).

316

As the data followed a normal distribution and had equal variances, we used an independent $\mathrm{t}$ test to compare groups. We applied a one-tailed t-test for the cognitive reappraisal subscale, as we predicted higher scores for the ASMR group. We used a two-tailed t-test for the expressive suppression subscale because we had no directional prediction for group differences.

\section{ASMR group differences in the Emotion Regulation Questionnaire (ERQ)}

The ASMR group showed significantly higher scores in the cognitive reappraisal subscale than the non-ASMR group $(t(134)=2.53, p<.01$, Cohen's $d=0.43$ ) (see Table 1). There were no significant differences between ASMR and non-ASMR groups for the expressive suppression subscale $(t(134)=-0.84, p=.40)$. These results can be observed in Figure 1 .

We also performed the same analysis using all the sample and, after including the gender of the participants as a predictor, obtaining similar results (see supplementary material)

\section{Table 1: ASMR and Non-ASMR scores in the Emotion Regulation Questionnaire.}

Figure 1: Plot of ASMR and Non-ASMR scores in the Emotion Regulation Questionnaire. Box-plot shows the scores of the two Emotional Regulation Questionnaire' subscales for the $\operatorname{ASMR}(\mathrm{n}=68)$ and non-ASMR $(\mathrm{n}=68)$ groups. Jittered points are individual subjects.

\section{Replicability of the effect using multiple random sub-samples.}

As our main analysis was performed on a subsample of 68 participants of the total sample who reported to experience ASMR (108 participants), we wanted to test the replicability and robustness of our effect by using multiple random sub-samples. Specifically, we repeated 1000 times the procedure of randomly choose subsamples, each of 68 participants of the total of 108 participants who reported experiencing ASMR. We compared each subsample with the 68 participants who reported not experiencing ASMR. Of these 1000 randomly chosen subsamples, we found that the ASMR group had significantly higher scores in the cognitive reappraisal subscale $84.60 \%$ of the time, binomial 95\% CI [82.18\%, 86.75\%]. Regarding the expressive suppression subscale, we found no significant differences in $97.90 \%$ of the subsamples, binomial 
341

342

343

344

345

346

347

348

349

350

351

352

353

354

355

356

357

358

359

360

361

362

363

364

365

366

367

368

369

370

371

372

373

374

375

376

377

378

379

380

95\% CI [96.75\%, 98.66\%]. In a high percentage of subsamples, we found results similar to those from our main analysis, supporting the replicability of the effect in different subsamples of our data.

\section{Differences in ERQ scores using ASMR self-reporters who sought out ASMR videos.}

Given that group selection was based on self-report over the internet after a brief description of the phenomenon, naïve people may confound the experience (for instance, with frisson) and erroneously report themselves as ASMR experiencers. In order to have a more accurate selection of participants who experience ASMR we performed the same analysis as before, but only with the subjects who report to experience ASMR and also sought ASMR videos before. This new subsample was composed of 58 participants (45 female, mean age $=22.59, S D=3.08$ ). We compared this new subsample with the 68 participants who reported not experiencing ASMR. Groups did not differ in age $(t(124)=1.50, p=.13)$ nor in the proportion of male to female participants $\left(\chi^{\wedge} 2(1)=0.09, p=.76\right)$.

In the cognitive reappraisal subscale, the ASMR self-reporters who have watched ASMR videos had marginally significant higher scores than non-ASMR subjects $(t(124)=1.66, p=.05$, Cohen's $d=0.30)$, with the ASMR group scoring $4.53(\mathrm{SD}=1.12)$ and the non-ASMR group scoring $4.20(\mathrm{SD}=1.16)$. In the expressive suppression subscale there were no significant differences between groups $(t(124)=-0.77, p=.44)$.

\section{ASMR-15 Score group differences}

We used the ASMR-15 Scale as a global corroboration that the selection of groups based on selfreport was different on several dimensions of the phenomenon. This analysis may help to confirm that participants who self-report to experience ASMR actually had felt it. Using the same sample from our main analysis, we wanted to test if participants who self-report to experience ASMR had higher scores in the four subscales of the ASMR-15, comparing with participants who do not report to experience ASMR.

Compared to the non ASMR group, the ASMR group had significantly higher scores in sensation $(t(134)=6.03, p<.001$, Cohen's $d=1.03)$, relaxation $(t(134)=5.20, p<.001$, Cohen's $d=$ $0.89)$, affect $(t(134)=5.08, p<.001$, Cohen's $d=0.87)$ and in altered consciousness $(t(134)$ $=3.59, p<.001$, Cohen's $d=0.62$ ). Additionally, using the ASMR-15 total score (the mean of the 15 items), the ASMR group had significantly higher scores than the non ASMR group (t(134) = $6.51, \mathrm{p}<.001$, Cohen's $\mathrm{d}=1.12$ ). These results indicate that participants who self-report as ASMR experiencers, also scores higher in different dimensions involved in experiencing ASMR, providing further support to the group selection. Descriptive values of the subscales can be found in the supplementary material.

\section{Discussion}

The present study investigated group differences between ASMR self-reporters and non-ASMR groups associated with emotional regulation strategies. We predicted that people who experience 
381

382

383

384

385

386

387

388

389

390

391

392

393

394

395

396

397

398

399

400

401

402

403

404

405

406

407

408

409

410

411

412

413

414

415

416

417

ASMR would show more significant use of cognitive reappraisal compared with non-ASMR self-reported controls. Our results confirmed our prediction, showing with a moderate effect size that participants who experience ASMR had higher scores in the cognitive reappraisal subscale of the emotion regulation questionnaire than the non-ASMR group. This finding suggests that the ASMR group uses cognitive reevaluation of the emotionally arousing situation to alter its emotional impact to a greater extent than the non-ASMR group. On the other hand, both groups showed similar scores in the other subscale of the same questionnaire, suggesting no group differences for the use of inhibition of emotion-expressive behavior.

Our finding supports the notion that ASMR is closely associated with emotional behavior rather than merely a sensory response. As we mentioned before, a growing body of studies has explored how ASMR elicits emotional arousal, which can be observed at different levels, such as psychological/behavioral (Barratt \& Davis, 2015), hemodynamical (Lochte et al., 2018), and electrophysiological (Fredborg, Champagne-Jorgensen, Desroches, \& Smith, 2021; Poerio et al., 2018). We now show that this phenomenon is also associated with strategies that allow us to control (regulate and manage) emotions when facing emotionally arousing situations. The higher use of the reappraisal strategy in people who experience ASMR is especially interesting given that reappraisal has been shown to be more effective to regulate emotions and related positively to well-being (Gross \& John, 2003; Morawetz et al., 2017). This contrasts with what has been shown for misophonia, suggested to be the opposite pole of ASMR, in which people have noticeable difficulties regulating emotions (Cassiello-Robbins et al., 2020).

We can speculate that the individual variations on reappraisal preferences might be associated with connectivity of brain networks associated with reappraisal, involving the orbitofrontal cortex selectively (Kanske, Heissler, Schönfelder, Bongers, \& Wessa, 2011). Studies in restingstate functional connectivity have reported that people who experience ASMR show the recruitment of the orbitofrontal cortex by sensory-motor networks, suggesting this is one neural substrate of ASMR's underlying emotional aspect. However, the differences reported in the present study may also be mediated by personality traits that have been independently related to ASMR and emotional regulation, such as neuroticism and openness to experience (Fredborg et al., 2017; McErlean \& Banissy, 2017; Morawetz et al., 2017; Wang et al., 2009). As we did not measure these traits in our sample, future investigations may explore the specific relations between ASMR, personality traits, and emotional regulation.

While we showed that ASMR self-reporters obtained higher cognitive reappraisal scores, our study cannot shed light on the mechanisms responsible for this association. Follow-up studies should specify the relationship between experiencing ASMR and cognitive reappraisal strategies to regulate one's emotional states. We hypothesize that the higher tendency of ASMR selfreporters to deploy cognitive reappraisal strategies is mediated by their capacity to be able to immerse themselves in different types of experiences (McErlean \& Banissy, 2017; Roberts et al., 
418

419

420

421

422

423

424

425

426

427

428

429

430

431

432

433

434

435

436

437

438

439

440

441

442

443

444

445

446

447

448

449

450

451

452

453

454

2019), a capacity that might give them greater flexibility to change the way they think about and re-appraise emotional situations. If this hypothesis is correct, constructs related to the capacity to be absorbed by imagery, such as fantasizing capabilities and daydreaming (Fox, Nijeboer, Solomonova, Domhoff, \& Christoff, 2013; Glisky et al., 1991), should mediate the relationship between ASMR and cognitive reappraisal.

In the present study, we also found that almost half of the ASMR sample (46\%) watch/listen to ASMR videos at least once a week (Figure S.4) which is in line with the reports of previous works, as in the case of Poerio et al., (2018) where 51\% reported watching videos daily or several times a week. This evidence suggests that a large proportion of the individuals who watched ASMR videos for the first time watch ASMR content again in a recurrent manner. Regarding the main motivations for watching ASMR videos, we found that relaxation was the most frequent reason for ASMR self-reporters followed by sleep induction (Figure S.5), which has been previously reported for people who experience ASMR (Barratt \& Davis, 2015). Interestingly, in our study, the people who do not experience ASMR and have watched ASMR videos also reported relaxation as the main motivation for watching ASMR content. This result may suggest that ASMR content may have the potential to help people to deal with anxiety regardless of whether they experience the tingles or not. However, previous works have shown this positive effect may occur only for individuals who experience ASMR (Poerio et al., 2018). Regarding the positive effect in sleep induction, ASMR triggers have been used to induce sleep in general population (Lee, Song, Shin, \& Lee, 2019). The main findings of the present study constitute a starting point to continue investigating on the underlying mechanism mediating the association between ASMR and emotional regulation, which in the future could also open the possibility to use ASMR videos/triggers as tools to promote emotion regulation strategies in general population.

We also used a multiple regression with gender as the first predictor and showed that gender did not play an essential role in our main finding (see supplementary material). This result suggests that individuals who experience ASMR report higher scores on cognitive reappraisal regardless of their gender. Nonetheless, we had a limited capacity to assess the indirect effect of gender on the results due to the sample was composed mostly of women (in the ASMR and non-ASMR, women made $75.36 \%$ of each group). This unbalance is a limitation of the present study reducing our capacity to reach a more informed conclusion about the potential gender effects. Curiously, this more extensive representation of women seems common in other ASMR studies with selfreporting subjects. In a study by McErlean and Banissy (2017), the percentage of women in the ASMR group was $69.88 \%$ and $80 \%$ in the non-ASMR group; in McErlean and Banissy (2018), the percentage of women was $62.5 \%$ in the ASMR group and $72.47 \%$ in the non-ASMR group. Finally, in the study by McErlean and Osborne-Ford (2020), the percentage of women was $74.19 \%$ in both groups. We believe that future studies on ASMR need to explore this tendency 
455

456

457

458

459

460

461

462

463

464

465

466

467

468

469

470

471

472

473

474

475

476

477

478

479

480

481

482

483

484

485

486

487

488

and also balance the proportion of female/male participants to precisely determine gender effects and potential gender differences in the experience of ASMR.

Finally, another limitation of the present study is related to the group selection's validity based on self-report over the internet. We grouped based on a YES/NO question widely used in previous studies (Barratt \& Davis, 2015; J. McErlean \& Banissy, 2018; Poerio et al., 2018; Valtakari, Hooge, Benjamins, \& Keizer, 2019). However, our description of the phenomenon was not very detailed (for instance, we did not provide examples of the triggers), potentially generating people confounding with other experiences, such as frisson. We then used complementary methods to corroborate that people who reported to experience ASMR actually did. We used the ASMR-15 scale, which has demonstrated effectiveness to measure the ASMR propensity in multiple dimensions of the phenomenon (Roberts et al., 2019), and also performed an additional analysis with only the people who sought ASMR videos. These results provided reliability to our group selection; however, this is still a big challenge to all ASMR studies performed over the internet. Some other methods have been used to ensure group selection is performed adequately (see ASMR checklist in Fredborg et al., 2017).

\section{Conclusions}

This is the first study that examines whether ASMR self-reporters show differences associated with emotional regulation strategies compared with non-ASMR controls. We showed that people who experience ASMR use the cognitive reappraisal strategy to a greater extent than non-ASMR people, suggesting more effectiveness in regulating emotions. The relevance of this finding relies on the fact that emotion regulation is fundamental for well-being, and this relationship between ASMR and emotional regulation may open the way to future research exploring the causal relationship between these features and also opening the possibility to use ASMR videos/triggers as tools to promote emotion regulation strategies, similar to how it is used to induce sleep (Lee et al., 2019).

Finally, our finding further elucidates individual differences related to this experience, supporting that ASMR is a real psychophysiological phenomenon associated with other psychological constructs and has remarkable consequences in affective/emotional dimensions and general well-being.

\section{Acknowledgements}

We want to thank Pilar Fajardo for her significant help setting the online questionnaires and organizing data. We also want to thank Gissella Di Giovanni (Gisse ASMR) and Abigail (Abi ASMR) for their kind support and help in spreading the study's information. 
489

490

491

492

493

494

495

496

497

498

499

500

501

502

503

504

505

506

507

508

509

510

511

512

513

514

515

516

517

518

519

520

521

522

523

524

525

\section{References}

Allaire, J. (2012). RStudio: integrated development environment for R. Boston, MA, 770, 394.

Barratt, E. L., \& Davis, N. J. (2015). Autonomous Sensory Meridian Response (ASMR): a flowlike mental state. PeerJ, 3, e851. https://doi.org/10.7717/peerj.851

Barratt, E. L., Spence, C., \& Davis, N. J. (2017). Sensory determinants of the autonomous sensory meridian response (ASMR): understanding the triggers. PeerJ, 5, e3846. https://doi.org/10.7717/peerj.3846

Cabello, R., Salguero, J. M., Fernández-Berrocal, P., \& Gross, J. J. (2013). A Spanish adaptation of the Emotion Regulation Questionnaire. European Journal of Psychological Assessment, 29(4), 234-240. https://doi.org/10.1027/1015-5759/a000150

Cassiello-Robbins, C., Anand, D., McMahon, K., Guetta, R., Trumbull, J., Kelley, L., \& Rosenthal, M. Z. (2020). The Mediating Role of Emotion Regulation Within the Relationship Between Neuroticism and Misophonia: A Preliminary Investigation. Frontiers in Psychiatry, 11, 1. https://doi.org/10.3389/fpsyt.2020.00847

Davis, M. H. (1983). Measuring individual differences in empathy: Evidence for a multidimensional approach. Journal of Personality and Social Psychology, 44(1), 113-126. https://doi.org/10.1037/0022-3514.44.1.113

del Campo, M. A., \& Kehle, T. J. (2016). Autonomous sensory meridian response (ASMR) and frisson: Mindfully induced sensory phenomena that promote happiness. International Journal of School and Educational Psychology, 4(2), 99-105. https://doi.org/10.1080/21683603.2016.1130582

Esménio, S., Soares, J. M., Oliveira-Silva, P., Zeidman, P., Razi, A., Gonçalves, Ó. F., ... Coutinho, J. (2019). Using resting-state DMN effective connectivity to characterize the neurofunctional architecture of empathy. Scientific Reports, 9(1). https://doi.org/10.1038/s41598-019-38801-6

Fox, K. C. R., Nijeboer, S., Solomonova, E., Domhoff, G. W., \& Christoff, K. (2013). Dreaming as mind wandering: evidence from functional neuroimaging and first-person content reports. Frontiers in Human Neuroscience, 7, 412.

Fredborg, B., Clark, J., \& Smith, S. D. (2017). An examination of personality traits associated with Autonomous Sensory Meridian Response (ASMR). Frontiers in Psychology, 8(FEB), 247. https://doi.org/10.3389/fpsyg.2017.00247

Fredborg, B. K., Champagne-Jorgensen, K., Desroches, A. S., \& Smith, S. D. (2021). An electroencephalographic examination of the autonomous sensory meridian response (ASMR). Consciousness and Cognition, 87. https://doi.org/10.1016/j.concog.2020.103053

Fredborg, B. K., Clark, J. M., \& Smith, S. D. (2018). Mindfulness and autonomous sensory meridian response (ASMR). PeerJ, 6, e5414. https://doi.org/10.7717/peerj.5414

Glisky, M. L., Tataryn, D. J., Tobias, B. A., Kihlstrom, J. F., \& McConkey, K. M. (1991). 
526

544

545

546

547

548

549

550

551

552

553

554

555

556

557

558

559

560

561

562

Absorption, Openness to Experience, and Hypnotizability. Journal of Personality and Social Psychology, 60(2), 263-272. https://doi.org/10.1037/0022-3514.60.2.263

Gross, J. J., \& John, O. P. (2003). Individual Differences in Two Emotion Regulation Processes: Implications for Affect, Relationships, and Well-Being. Journal of Personality and Social Psychology, 85(2), 348-362. https://doi.org/10.1037/0022-3514.85.2.348

Harrison, L., \& Loui, P. (2014). Thrills, chills, frissons, and skin orgasms: Toward an integrative model of transcendent psychophysiological experiences in music. Frontiers in Psychology, 5(JUL), 1-6. https://doi.org/10.3389/fpsyg.2014.00790

John, O. P., Naumann, L. P., \& Soto, C. J. (2008). Paradigm shift to the integrative Big Five Trait taxonomy: History, measurement, and conceptual issues. In Handbook of personality: Theory and research (pp. 114-158). Retrieved from https://psycnet.apa.org/record/200811667-004

Kumar, S., Tansley-Hancock, O., Sedley, W., Winston, J. S., Callaghan, M. F., Allen, M., ... Griffiths, T. D. (2017). The Brain Basis for Misophonia. Current Biology, 27(4), 527-533. https://doi.org/10.1016/J.CUB.2016.12.048

Laghi, F., Lonigro, A., Pallini, S., \& Baiocco, R. (2018). Emotion Regulation and Empathy: Which Relation with Social Conduct? Journal of Genetic Psychology, 179(2), 62-70. https://doi.org/10.1080/00221325.2018.1424705

Lee, M., Song, C.-B., Shin, G.-H., \& Lee, S.-W. (2019). Possible Effect of Binaural Beat Combined With Autonomous Sensory Meridian Response for Inducing Sleep. Frontiers in Human Neuroscience, 13. https://doi.org/10.3389/fnhum.2019.00425

Lochte, B. C., Guillory, S. A., Richard, C. A. H., \& Kelley, W. M. (2018). An fMRI investigation of the neural correlates underlying the autonomous sensory meridian response (ASMR). BioImpacts, 8(4), 295-304. https://doi.org/10.15171/bi.2018.32

Lockwood, P. L., Seara-Cardoso, A., \& Viding, E. (2014). Emotion regulation moderates the association between empathy and prosocial behavior. PLOS ONE, 9(5). https://doi.org/10.1371/journal.pone.0096555

McErlean, A. B. J., \& Banissy, M. J. (2017). Assessing individual variation in personality and empathy traits in self-reported autonomous sensory meridian response. Multisensory Research, 30(6), 601-613. https://doi.org/10.1163/22134808-00002571

McErlean, A. B. J., \& Osborne-Ford, E. J. (2020). Increased absorption in autonomous sensory meridian response. PeerJ, 2020(2). https://doi.org/10.7717/peerj.8588

McErlean, J., \& Banissy, M. J. (2018). Increased misophonia in self-reported Autonomous Sensory Meridian Response. PeerJ, 6, e5351. https://doi.org/10.7717/peerj.5351

Morawetz, C., Alexandrowicz, R. W., \& Heekeren, H. R. (2017). Successful emotion regulation is predicted by amygdala activity and aspects of personality: A latent variable approach. Emotion, 17(3), 421-441. https://doi.org/10.1037/emo0000215 
563

564

565

566

567

568

569

570

571

572

573

574

575

576

577

578

579

580

581

582

583

584

585

586

587

588

589

590

591

592

593

594

595

596

597

598

599

Morris, M. (2018). A Simply Marvellous Reaction: Autonomous Sensory Meridian Response and the Desk Tutorial. Architectural Design, 88(2), 50-57. https://doi.org/10.1002/ad.2279

Nelson, B. D., Fitzgerald, D. A., Klumpp, H., Shankman, S. A., \& Phan, K. L. (2015). Prefrontal engagement by cognitive reappraisal of negative faces. Behavioural Brain Research, 279, 218-225. https://doi.org/10.1016/j.bbr.2014.11.034

Poerio, Blakey, E., Hostler, T. J., \& Veltri, T. (2018). More than a feeling: Autonomous sensory meridian response (asmr) is characterized by reliable changes in affect and physiology. PLoS ONE, 13(6), e0196645. https://doi.org/10.1371/journal.pone.0196645

Poerio, G. (2016). Could Insomnia Be Relieved with a YouTube Video? The Relaxation and Calm of ASMR. In The Restless Compendium (pp. 119-128). Cham: Springer International Publishing. https://doi.org/10.1007/978-3-319-45264-7_15

Revelle, W., \& Revelle, M. W. (2015). Package 'psych.' The Comprehensive R Archive Network.

Roberts, N., Beath, A., \& Boag, S. (2019). Autonomous sensory meridian response: Scale development and personality correlates. Psychology of Consciousness: Theory Research, and Practice, 6(1), 22-39. https://doi.org/10.1037/cns0000168

Roberts, N., Beath, A., \& Boag, S. (2020). Autonomous Sensory Meridian Response: Sensitivity and Personality Correlates. Journal of Individual Differences. https://doi.org/10.1027/16140001/a000337

Rouw, R., \& Erfanian, M. (2018). A Large-Scale Study of Misophonia. Journal of Clinical Psychology. https://doi.org/10.1002/jclp.22500

Salimpoor, V. N., Benovoy, M., Larcher, K., Dagher, A., \& Zatorre, R. J. (2011). Anatomically distinct dopamine release during anticipation and experience of peak emotion to music, 14(2). https://doi.org/10.1038/nn.2726

Smith, S. D., Fredborg, B. K., \& Kornelsen, J. (2019). Atypical Functional Connectivity Associated with Autonomous Sensory Meridian Response: An Examination of Five Resting-State Networks. Brain Connectivity, brain.2018.0618. https://doi.org/10.1089/brain.2018.0618

Smith, S. D., Katherine Fredborg, B., \& Kornelsen, J. (2017). An examination of the default mode network in individuals with autonomous sensory meridian response (ASMR). Social Neuroscience, 12(4), 361-365. https://doi.org/10.1080/17470919.2016.1188851

Tabachnick, B. G., \& Fidell, L. S. (2013). Using multivariate statistics. Boston: Pearson Education.

Tang, Y. Y., Tang, R., \& Posner, M. I. (2016, June 1). Mindfulness meditation improves emotion regulation and reduces drug abuse. Drug and Alcohol Dependence. Elsevier Ireland Ltd. https://doi.org/10.1016/j.drugalcdep.2015.11.041

Valero-Mora, P. M. (2010). ggplot2: Elegant Graphics for Data Analysis . Journal of Statistical Software (Vol. 35). springer. https://doi.org/10.18637/jss.v035.b01

PeerJ reviewing PDF | (2020:12:56562:2:0:NEW 15 Apr 2021) 
600

601

602

603

604

605

606

607
Valtakari, N. V., Hooge, I. T. C., Benjamins, J. S., \& Keizer, A. (2019). An eye-tracking approach to Autonomous sensory meridian response (ASMR): The physiology and nature of tingles in relation to the pupil. PLOS ONE, 14(12), e0226692. https://doi.org/10.1371/journal.pone.0226692

Wang, L., Shi, Z., \& Li, H. (2009). Neuroticism, extraversion, emotion regulation, negative affect and positive affect: The mediating roles of reappraisal and suppression. Social Behavior and Personality, 37(2), 193-194. https://doi.org/10.2224/sbp.2009.37.2.193 
Figure 1

Plot of ASMR and Non-ASMR scores in the Emotion Regulation Questionnaire.

Box-plot shows the scores of the two Emotional Regulation Questionnaire' subscales for the ASMR $(n=68)$ and non-ASMR $(n=68)$ groups. Jittered points are individual subjects.

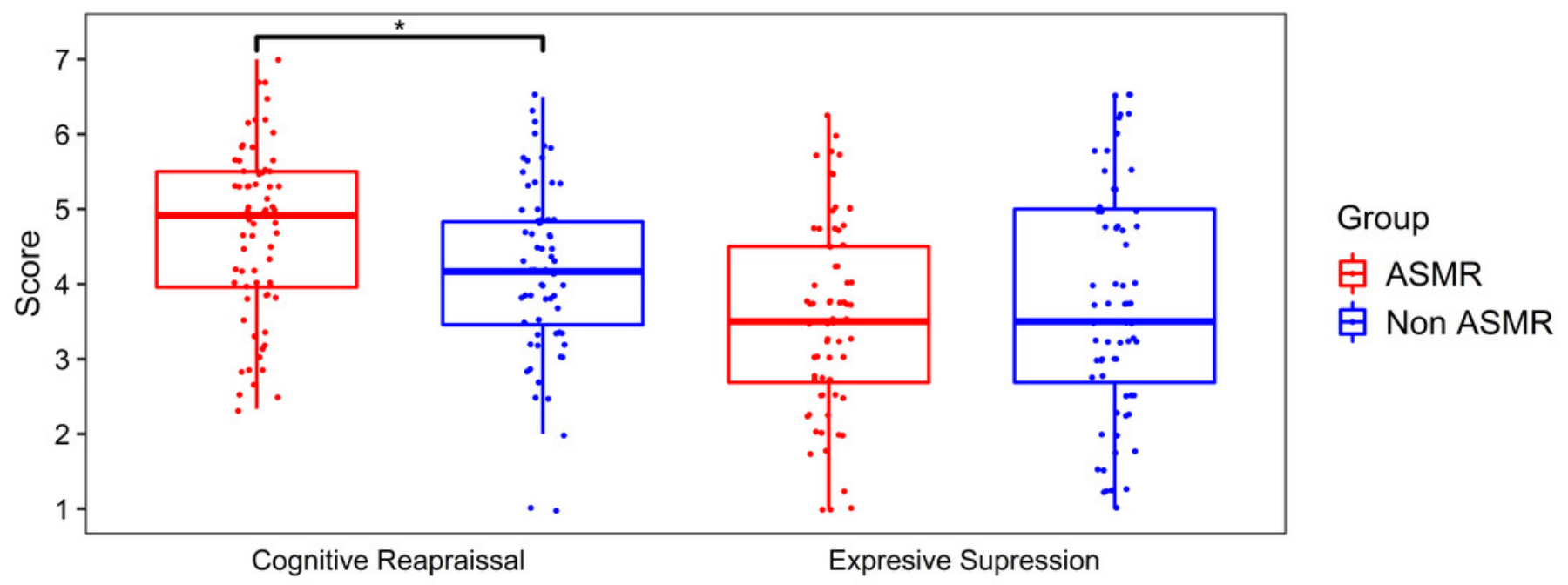




\section{Table 1 (on next page)}

ASMR and Non-ASMR scores in the Emotion Regulation Questionnaire. 
1 Table 1: ASMR and Non ASMR scores in the Emotion Regulation Questionnaire.

\begin{tabular}{|c|c|c|c|c|c|c|}
\hline \multirow{3}{*}{ Scale } & \multicolumn{6}{|c|}{ Group } \\
\hline & \multicolumn{3}{|c|}{ ASMR } & \multicolumn{3}{|c|}{ Non ASMR } \\
\hline & Mean & Median & SD & Mean & Median & SD \\
\hline Cognitive & 4.69 & 4.92 & 1.14 & 4.19 & 4.17 & 1.16 \\
\hline \multicolumn{7}{|l|}{ Reappraisal } \\
\hline Expressive & 3.53 & 3.50 & 1.28 & 3.73 & 3.5 & 1.51 \\
\hline Suppression & & & & & & \\
\hline
\end{tabular}

2 